\title{
Research advances in Dieulafoy's disease of the bronchus (Review)
}

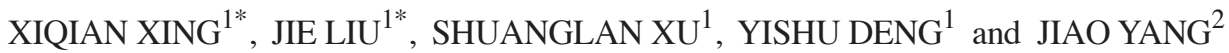 \\ ${ }^{1}$ Department of Respiratory Medicine, The Affiliated Hospital of Yunnan University, The Second People's Hospital of \\ Yunnan Province, Kunming, Yunnan 650021; ${ }^{2}$ First Department of Respiratory Medicine, \\ First Affiliated Hospital of Kunming Medical University, \\ Kunming, Yunnan 650032, P.R. China
}

Received January 16, 2019; Accepted July 14, 2020

DOI: $10.3892 /$ etm.2021.11023

\begin{abstract}
Dieulafoy's disease is characterized by abnormal submucosal arteries and results in acute luminal hemorrhage. Dieulafoy's lesions can also be found in the submucosa of the bronchus. Due to its low incidence rate and non-specific clinical symptoms, Dieulafoy's disease is easy to overlook, but can lead to massive bleeding and high rates of mortality. Therefore, improvements in the understanding of the disease are necessary. The awareness of the disease and associated diagnostic and treatment techniques have continued to improve, and thus, an increasing number of cases of Dieulafoy's disease of the bronchus have been reported. In the present review, 74 cases of Dieulafoy's disease are summarized. New technologies such as endobronchial ultrasound, narrow-band imaging, angiography and argon plasma treatment have been found to be increasingly applied to diagnose and treat Dieulafoy's disease of the bronchus. Therefore, the primary focus of this systematic review is to highlight advances in the diagnosis and treatment of bronchial Dieulafoy's disease.
\end{abstract}

Correspondence to: Professor Yishu Deng, Department of Respiratory Medicine, The Affiliated Hospital of Yunnan University, The Second People's Hospital of Yunnan Province, 176 Qing Nian Road, Kunming, Yunnan 650021, P.R. China

E-mail: dengyishukm@163.com

Professor Jiao Yang, First Department of Respiratory Medicine, First Affiliated Hospital of Kunming Medical University, 295 Xichang Road, Kunming, Yunnan 650032, P.R. China

E-mail: yangjiaokmu@yahoo.com

${ }^{*}$ Contributed equally

Abbreviations: CT, computed tomography; EBUS, endobronchial ultrasonography; SBAE, selective bronchial artery embolization

Key words: Dieulafoy's disease, bronchi, diagnosis, treatment, outcome

\section{Contents}

1. Introduction

2. Methods

3. Pathogenesis

4. Incidence

5. Lesion site

6. Clinical manifestations

7. Auxiliary examination

8. Diagnosis

9. Differential diagnosis

10. Treatment and prognosis

11. Conclusions

\section{Introduction}

Dieulafoy's disease, initially described as 'exulceratio simplex' (1) in 1897, is an acute luminal hemorrhage caused by the rupture of a feeding artery under the actions of external factors, or spontaneous rupture owing to vascular malformations of the gastrointestinal, biliary or bronchial wall; the feeding artery does not taper to capillaries after entering the submucosa, but remains constant in diameter and protrudes from the intestinal lumen. After $>100$ years of revisions and improved understanding, the disease was renamed as Dieulafoy's disease.

Dieulafoy's disease of the bronchus was first described by Sweerts et al (2) in 1995, and to date, <100 cases have been reported worldwide. However, as the condition is under-recognized and frequently underdiagnosed, the actual incidence is likely to be much higher. Therefore, it is necessary to increase our awareness of Dieulafoy's disease of the bronchus, which is considered to be one of the primary causes of massive hemoptysis (3). Although the natural history, diagnosis and preferred treatment of the disease are still unclear, Dieulafoy's disease of the bronchus is believed to be caused by both congenital (2) and acquired factors (4). In previous years, the awareness of the disease and associated techniques of diagnosis and treatment have continued to improve, and novel diagnostic technologies have been increasingly applied. The present review summarizes the research advances in Dieulafoy's disease of the bronchus. 


\section{Methods}

The terms 'bronchial OR bronchus' AND 'dieulafoy OR dieulafoy's' were searched in the PubMed and Embase databases, covering the period between January 1985 and December 2019. Literature associated with a definitive diagnosis or a high suspicion of Dieulafoy's disease of the bronchus was screened, with abstracts of meetings excluded. There were no limitations on the language of the publications. A manual search was then conducted according to the reference lists of the published articles.

\section{Pathogenesis}

Dieulafoy's disease of the bronchus is pathologically characterized by the rupture and bleeding of a dilated or abnormal artery in the bronchial submucosa. The dilated or abnormal artery passes through the bronchial wall next to the bronchial cavity, and is surrounded by a thin mucosal epithelial layer. Abnormal vessels predominantly branch from the bronchial artery system and rarely from the pulmonary artery (5).

The pathogenesis of bronchial Dieulafoy's disease remains to be clarified. Most researchers believe that the disease is congenital, while others believe that it is acquired or is simply an abnormality of normal blood vessels (2,4-6). The etiology and pathogenesis of Dieulafoy's disease of the bronchus may be associated with congenital abnormalities of bronchial and pulmonary arteries, chronic inflammation or injury of the airway, and is also considered to be associated with long-term heavy smoking (7). Almost half of the patients are smoking or had smoked in our study (31 of the 74 patients had a history of smoking). Parrot et al (7) reported the cases of seven patients with Dieulafoy's disease of the bronchus, all of whom were long-term heavy smokers (mean smoking capacity, 49 packs/year), which supports the aforementioned findings. Moreover, some patients had a history of tuberculosis (6 of the 74 patients developed tuberculosis), indicating a possible association with inflammatory injury in tuberculosis or stretching and dilation of the bronchial artery (7). In addition, 15 patients developed other respiratory diseases and two patients had a history of cardiovascular disease (8). These findings indicate that Dieulafoy's disease of the bronchus may be associated with a history of basic diseases, especially respiratory diseases such as tuberculosis (9-12), pneumonia $(13,14)$ and bronchiectasis $(15)$.

\section{Incidence}

In total, 74 cases of bronchial Dieulafoy's disease have been identified in the past 20 years since the first reported case in 1995 (2,4-44). Owing to the substantially low incidence of Dieulafoy's disease of the bronchus, there have been no statistical reports of its exact incidence. Among the patients reported, the youngest was 5 years old, and the oldest was 85 years old. Subjects aged 30-70 years were at a high risk, accounting for $80 \%$ of the total patients (Table I). The male-to-female incidence ratio was $\sim 2: 1$ (45 male vs. 24 female patients), although the reason for the sex differences in Dieulafoy's disease of the bronchus is not clear.

\section{Lesion site}

A total of 74 cases of Dieulafoy's disease of the bronchus were summarized, including 48 in the right bronchus (19 in the right lower lobe, 12 in the right middle lobe, 9 in the right upper lobe, 5 in the right lower and middle lobes, 2 in the right middle and upper lobes, and 1 in the entire right lung), 20 in the left bronchus (11 in the left lower lobe, 1 in the left main bronchus, 7 in the left upper lobe, and 1 in the left lung), 1 in the lingula, and 6 in an unspecified location. An analysis of the reported cases revealed that Dieulafoy's disease of the bronchus commonly occurs in the right bronchus, and that patients with lesions in the right bronchus account for approximately two-thirds of the total cases. These differences may be due to the anatomical characteristics of the bronchi. The right bronchus is short and thick so that foreign bodies are more likely to enter and cause infection, one of the potential causes of Dieulafoy's disease of the bronchus. Therefore, biopsies should be performed with caution to prevent hemorrhage in patients with cryptogenic hemoptysis if a lesion (especially in the right bronchus) with similar manifestations to Dieulafoy's disease is demonstrated by bronchoscopy.

\section{Clinical manifestations}

Recurrent hemoptysis is a common symptom of Dieulafoy's disease of the bronchus. The maximum amount of hemoptysis has been reported as 2,000 $\mathrm{ml}$, and is often without an obvious cause (14). A previously reported patient with Dieulafoy's disease presented with chest pain and no hemoptysis; the latter only occurred after bronchoscopic biopsy, and a definitive diagnosis was established by bronchial angiography (39). Indeed, patients with Dieulafoy's disease frequently visit the hospital with a cough $(12,36)$, infection $(12,20)$ or respiratory failure $(33,34)$. In conclusion, the clinical symptoms of Dieulafoy's disease of the bronchus are non-specific. Therefore, physicians should not only pay more attention to patients with massive hemoptysis, but also focus on patients with recurrent respiratory symptoms.

\section{Auxiliary examination}

In Dieulafoy's disease of the bronchus, chest X-rays and computed tomography (CT) scans are rarely positive for symptoms other than the manifestation of an intrapulmonary hemorrhage and the original lung disease. Only a few cases of endobronchial nodules have been identified by chest CT examination (17). By contrast, multi-slice CT angiography can clearly indicate the shape and direction of bronchial pulmonary vessels, which may detect a tortuous and dilated bronchial artery (22), and may also demonstrate well-enhanced endobronchial nodules (25).

Owing to cryptogenic hemoptysis, most patients who undergo bronchoscopic examination and bronchoscopy demonstrate massive endobronchial hemorrhage, which may even be accompanied by blood clot formation (12,36). Mucosal nodular projections can be a few millimeters in diameter and height with a smooth surface (36). The lesion may also be congested and rough, with slight vascular pulsation in specific cases. Sometimes the protruding surface is coated with yellow-white exudate forming a 'little white hat'-like shape, easily misdiagnosed as an endobronchial tumor nodule (7) (Fig. 1). The nodule may show as a neoplasm-like granulation nodule, leading to 


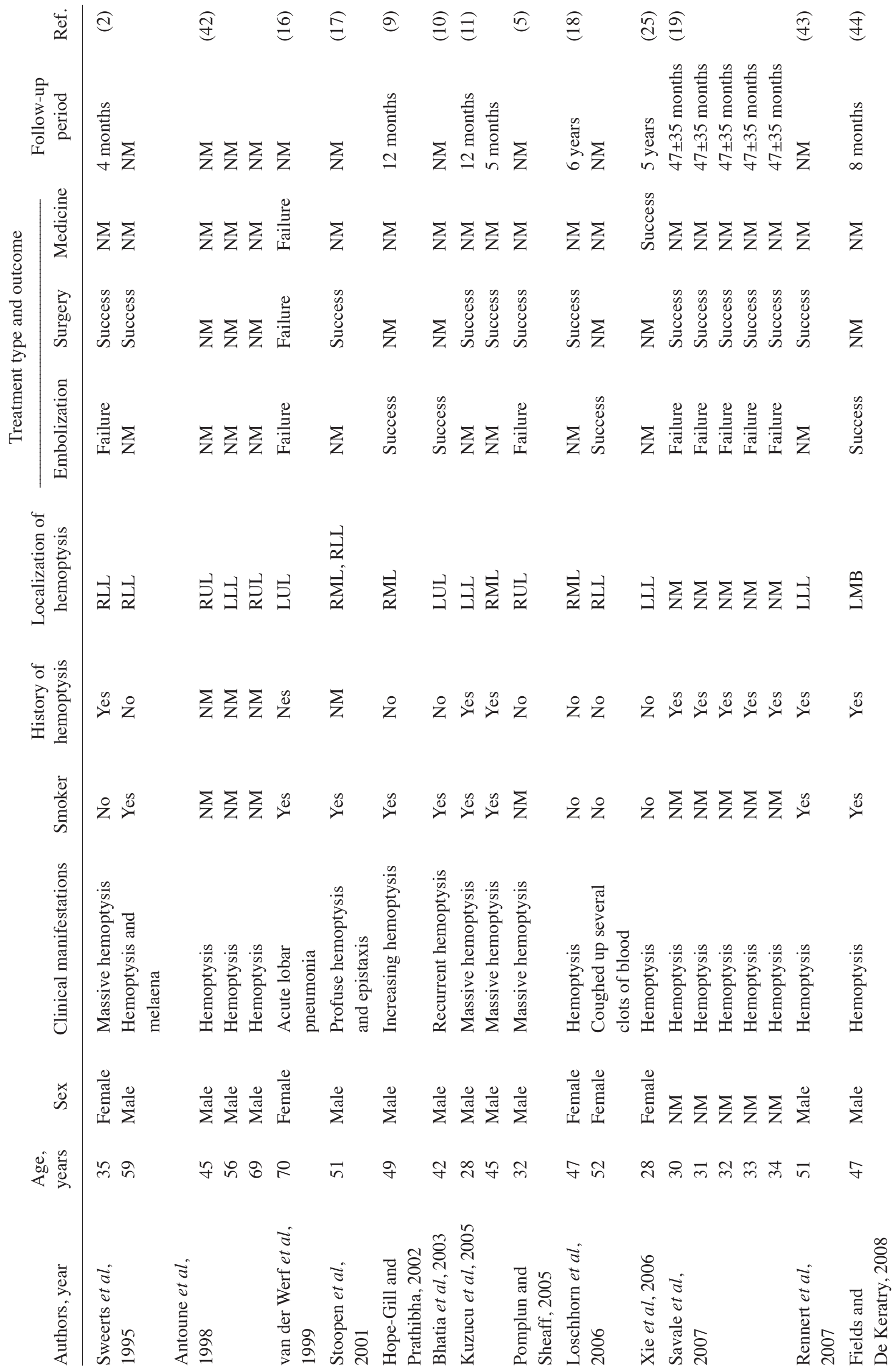




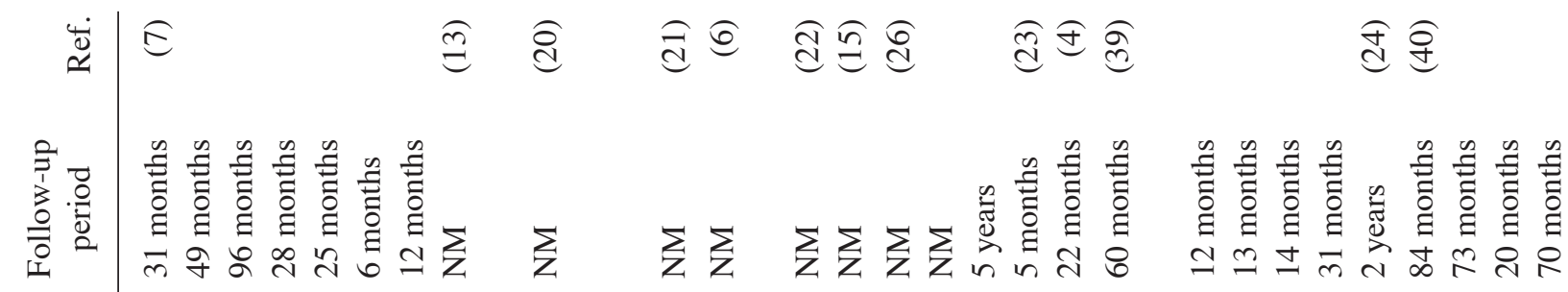

范

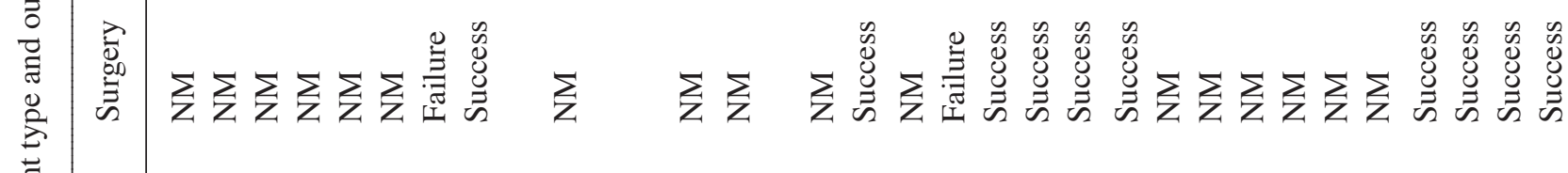

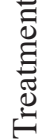

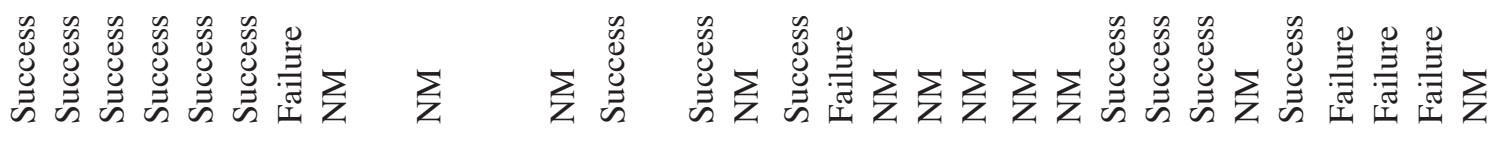

4

ธี $\frac{n}{n}$

荧

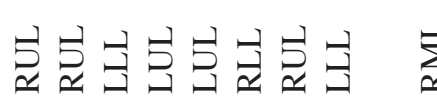

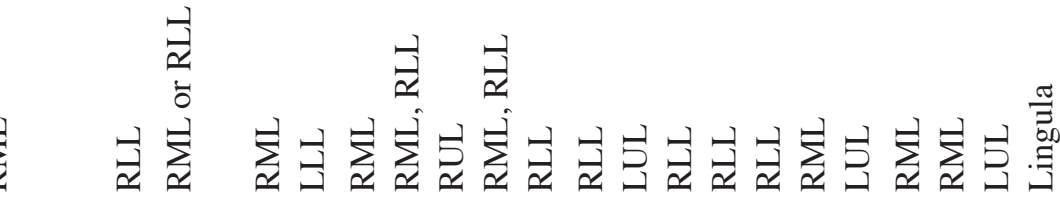

$\overrightarrow{\vec{x}} \overrightarrow{\vec{x}}$

t)

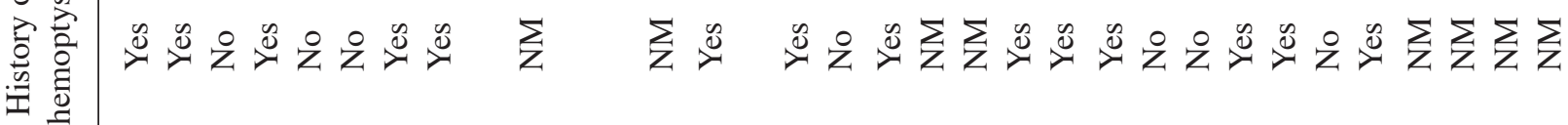

苛

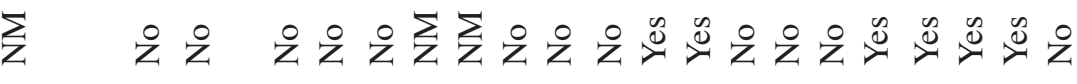

$\cdot \frac{n}{n} \cdot \frac{n}{n} \cdot \frac{n}{n} \cdot \frac{n}{n} \cdot \frac{n}{n} \cdot \frac{n}{n} \cdot \frac{n}{n}$

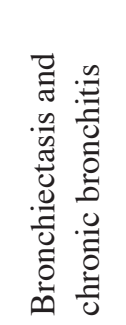

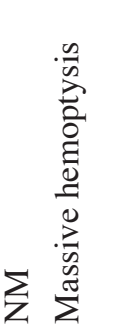

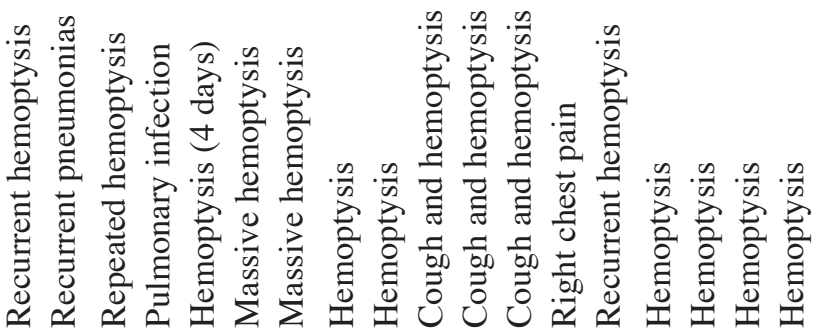

๘

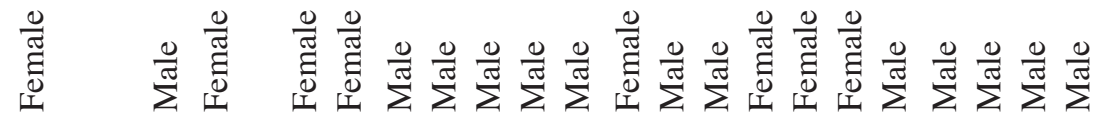

$\underset{\substack{0 \\ \hdashline}}{\infty}$

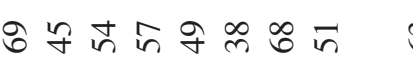

6.

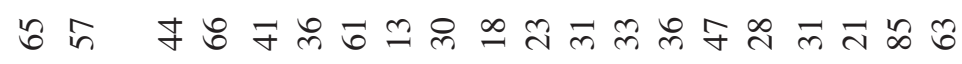




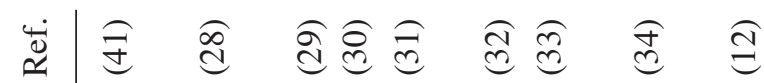

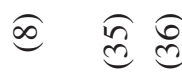

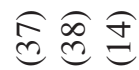

F.

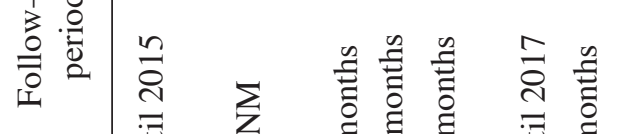

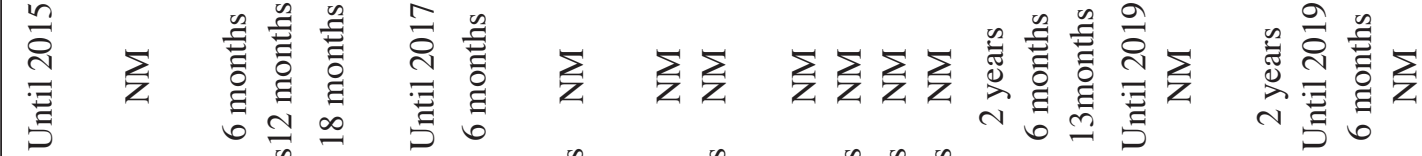

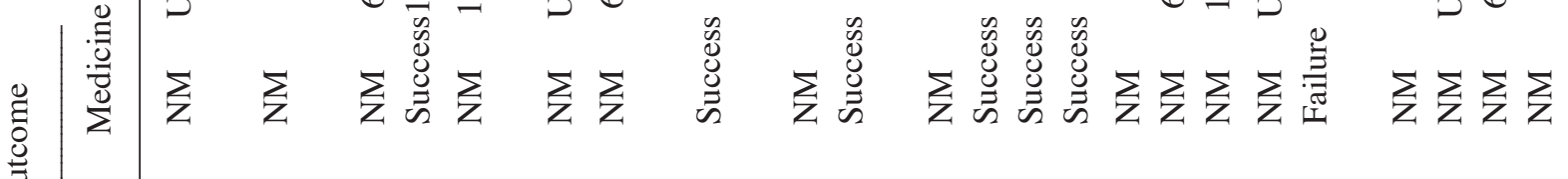

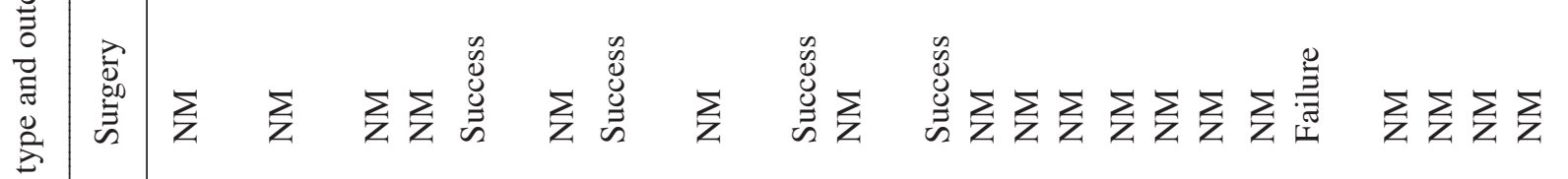

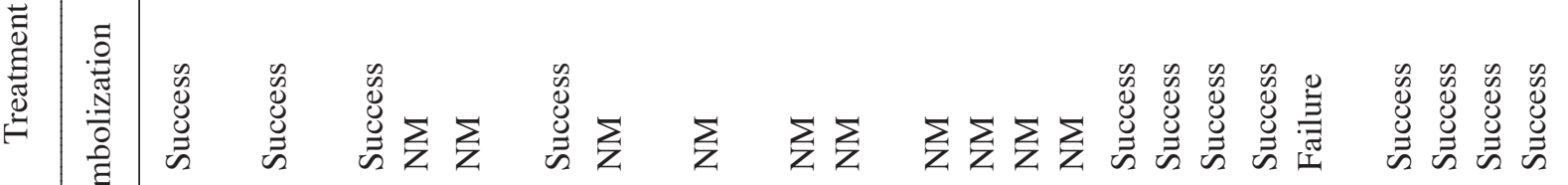

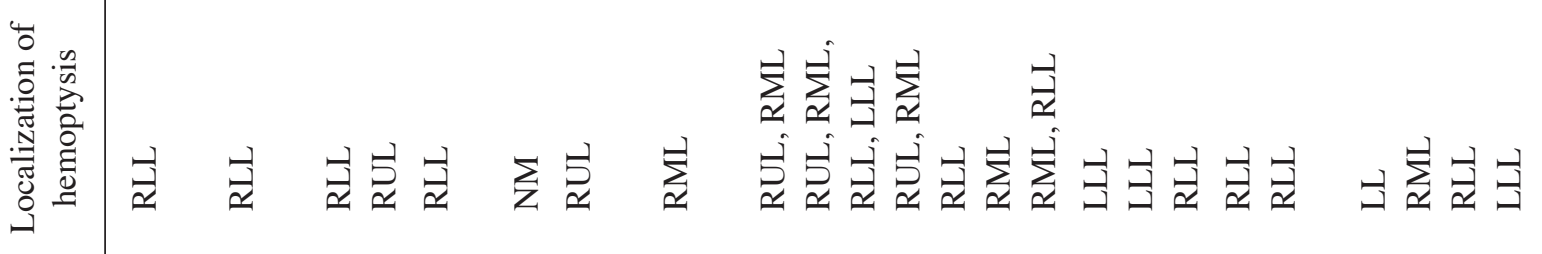

$\leftarrow \cdot \frac{n}{0}$

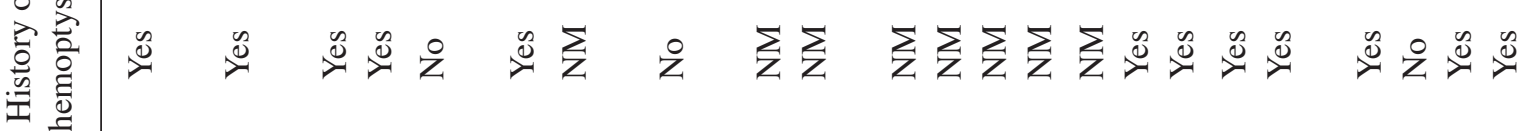

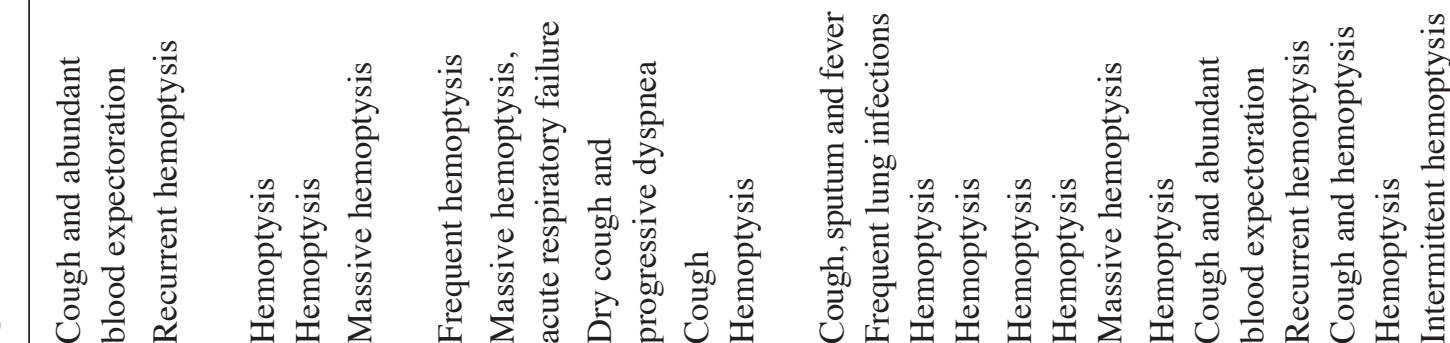

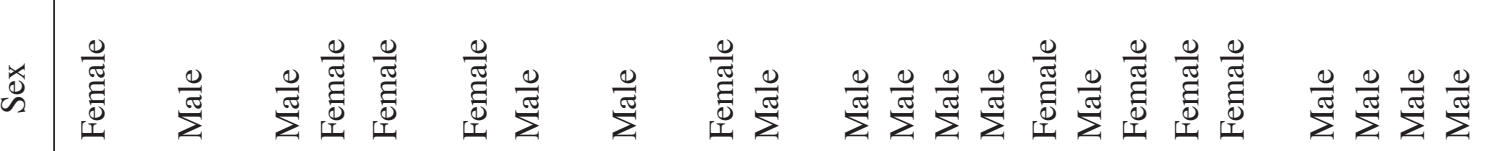

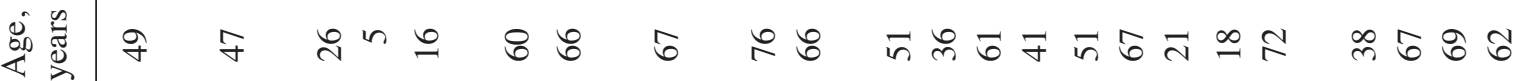




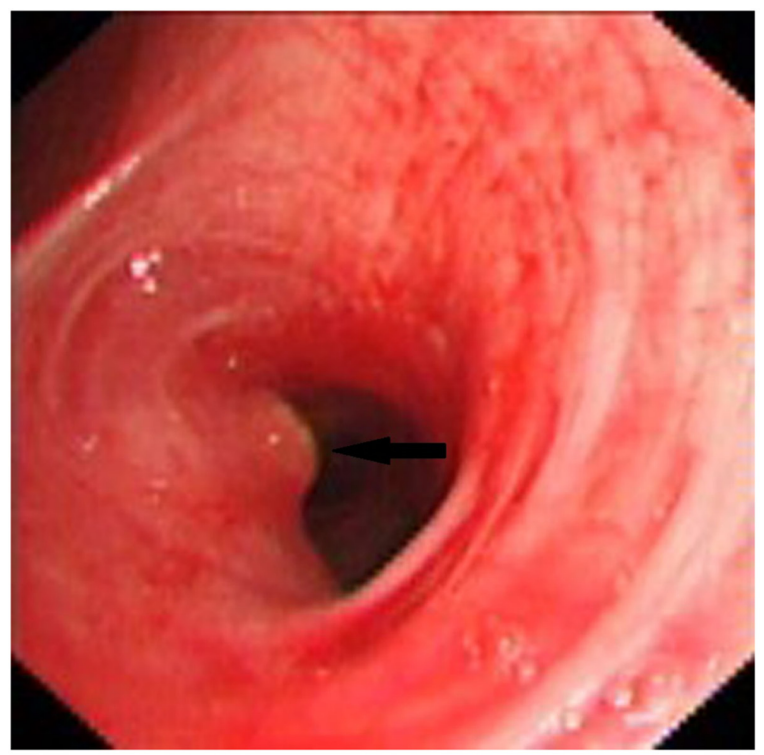

Figure 1. Small nodular protrusion from the mucosa of the left lingual segment of the bronchus. Protrusion of $\sim 3 \mathrm{~mm}$ in diameter and height, coated with yellow-white exudate, and forming a 'little while hat' (black arrow).

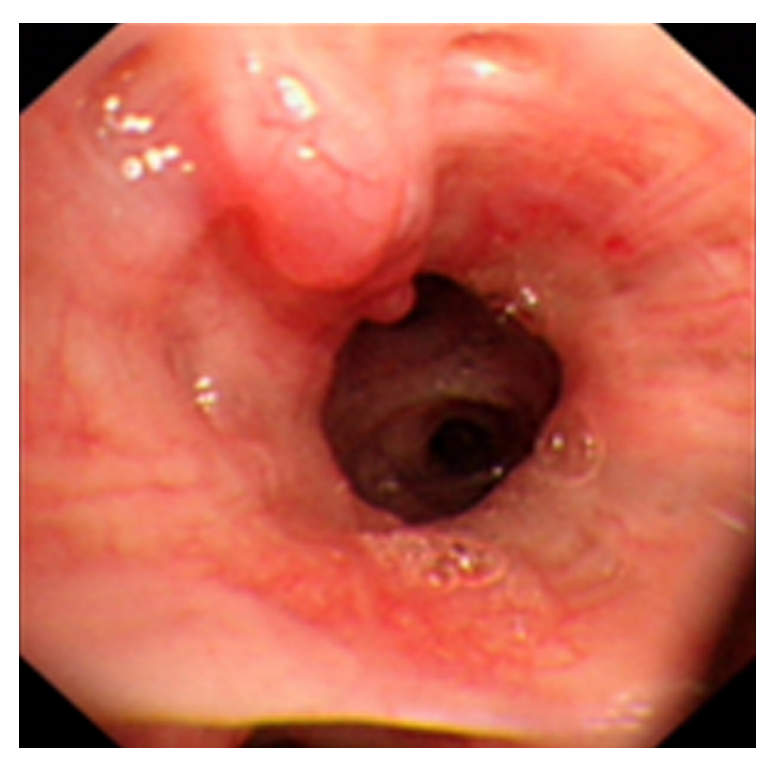

Figure 2. Abnormal vessels are tortuous and dilated, with purple earthworm-like alterations (black arrow) in the submucosa of the posterior basal segmental bronchus of the right lower lobe.

local obstruction of the bronchial lumen and causing obstructive pneumonia. If the nodule is mistaken for a neoplasm, subsequent biopsy may lead to a large hemorrhage and death by suffocation. Abnormal vessels in the submucosa can be tortuous and dilated, and with a worm-like shape (Fig. 2), sometimes with fork-like 'twigs'. This type of case is easily mistaken for submucosal tumor infiltration, and subsequent biopsies may lead to fatal hemorrhage. As the bronchial cavity is filled with blood and blood clots, it is difficult to find a small mucosal protrusion, or the mucosal protrusion is localized below the subsegmental bronchus and thus cannot be seen by conventional bronchoscopy.

Bronchial angiography contributes to the diagnosis of Dieulafoy's disease of the bronchus. Bronchial angiography

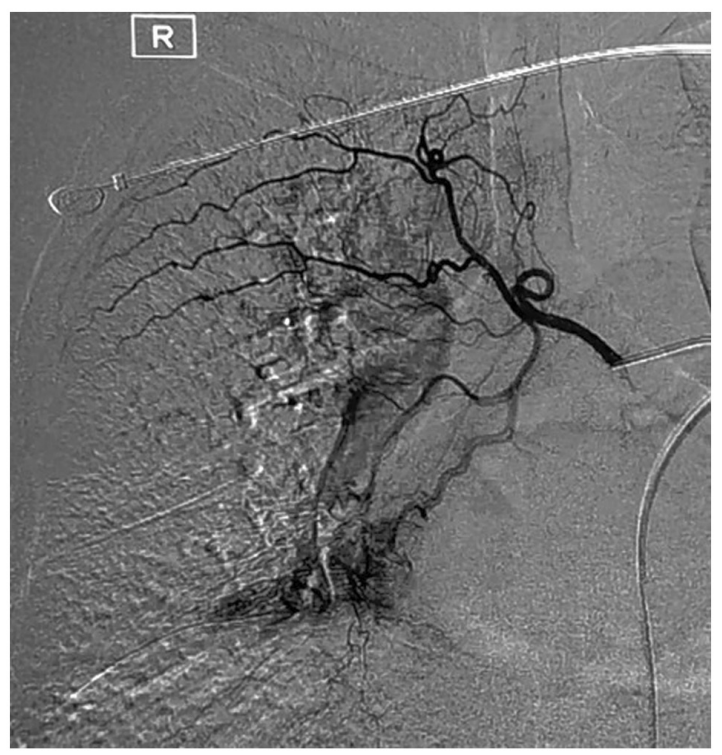

Figure 3. Thickened and disordered lower right bronchial artery shown by bronchial angiography.

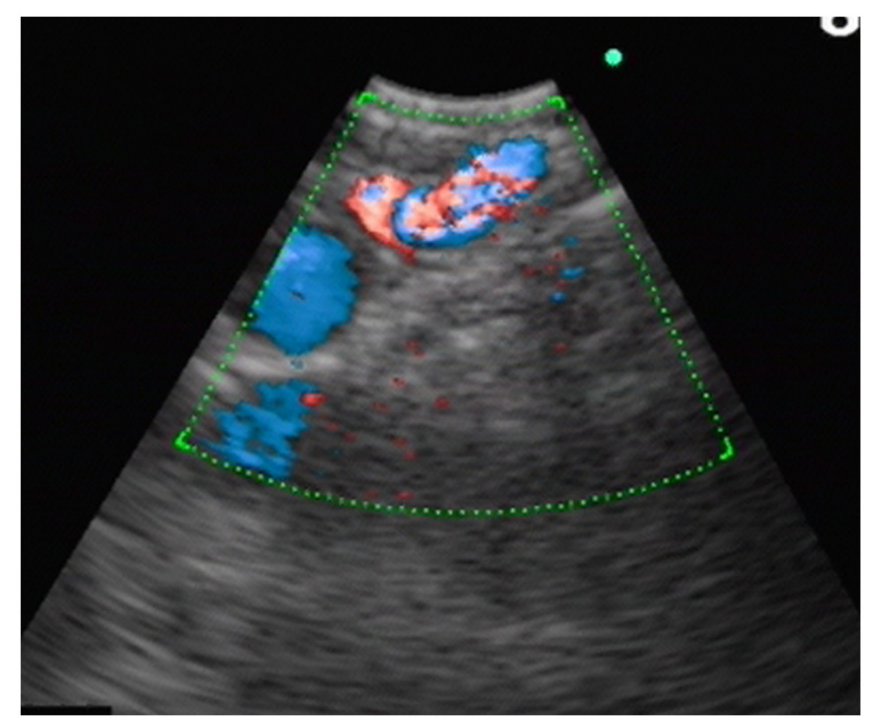

Figure 4. A 1.5 -mm diameter small blood vessel in a submucosal lesion examined by endobronchial ultrasound in color Doppler mode.

shows a rich blood supply in the corresponding site of the lesion (23); the bronchial artery is tortuous, dilated and deformed, with signs of bleeding (Fig. 3).

An endobronchial ultrasound can be used to clarify the nature of any endobronchial protrusion. This technique helps to clarify the nature of the nodular lesion and provides clues for disease diagnosis. The major manifestation is a fluid echo-free zone in the submucosal lesion, and the Doppler mode can be used to detect blood flow (21) (Fig. 4).

\section{Diagnosis}

The possibility of Dieulafoy's disease of the bronchus should be considered in patients with hemoptysis when chest X-ray and CT examinations demonstrate no obvious abnormalities 
other than pulmonary hemorrhage. Dieulafoy's disease of the bronchus is largely diagnosed according to the presentations of bronchoscopy, bronchial angiography and pathology of surgical or autopsy specimens. Numerous researchers consider that pathological examination of biopsies, surgical or autopsy specimens is required for a definite diagnosis. However, there are no uniform diagnostic criteria, and since pathological biopsies can lead to fatal hemorrhages, the need for pathological diagnosis remains controversial. In some cases, the diagnosis is based on the manifestations revealed by bronchoscopy and bronchial angiography $(24,25,39)$. Endobronchial ultrasonography (EBUS) is a new diagnostic method used to detect the lesions of Dieulafoy's disease (21).

Bronchoscopy. Under bronchoscopy, a mucosal protrusion a few millimeters in diameter and height is visible in the corresponding site of the bleeding bronchus. On the top of the protrusion, the mucosa turns white without a pulsating sensation; the surrounding mucosa may be normal or slightly congested. In some cases, abnormal blood vessels within the submucosa are tortuous and dilated in an earthworm-like pattern, sometimes presenting with a purple nodular shape (39). To prevent uncontrollable hemorrhage, caution must be taken during biopsy if Dieulafoy's disease is suspected (based on the results of bronchoscopy). Yang et al (26) analyzed the clinical data of 22 patients with Dieulafoy's disease, including 12 with hemorrhage during biopsy. Bleeding stopped in eight patients after local hemostasis, and in two after lobectomy of the diseased lobe; another two patients died of massive hemorrhage. The remaining 10 patients did not undergo bronchoscopic biopsy. Our previous study reported the cases of six patients, including two undergoing bronchoscopic biopsy, both of whom suffered hemorrhage; the maximum amount of hemorrhage was $\sim 1,000 \mathrm{ml}$, and bleeding stopped in both cases after selective bronchial artery embolization (39). Since 2014, with an improvement of the understanding of Dieulafoy's disease, biopsy has been avoided for nodules suspected to be caused by Dieulafoy's disease in the trachea $(23,41)$, which has effectively reduced the probability of massive hemorrhage in Dieulafoy's disease.

Bronchial angiography. As aforementioned, bronchial angiography can be used to indicate the rich blood supply to the corresponding site of the lesion. The bronchial artery is tortuous, dilated and deformed, with signs of bleeding (39).

Multi-slice CT angiography of the bronchial or pulmonary artery. This technique is used to visualize an abnormal bronchial artery associated with tortuosity and dilation (22), and sometimes detects well-enhanced endobronchial nodules (25). A CT value of $>100$ for the enhanced lesion should be considered to indicate a vascular lesion.

Endobronchial ultrasound. When convex probe EBUS is used to detect blood flow within a lesion, it often shows a fluid echo-free zone in submucosal lesions; the blood flow can be displayed in the color or energy Doppler mode (21). Owing to its large diameter, convex probe EBUS cannot reach the upper lobe bronchus or segmental bronchus. Alternatively, radial probe EBUS can be used to examine the lesion. However, the latter approach has no Doppler mode and thus cannot determine blood flow within the lesion. Nonetheless, radial probe EBUS can indicate an echo-free zone, which may be considered for vascular lesions in patients with hemoptysis.

Narrow-band imaging. Wang et al (45) reported that narrow band imaging (NBI) can display endobronchial lesions such as bronchial artery-pulmonary artery fistulae. Thickened blood vessels and capillaries are tortuous and disordered in the submucosa of the lesion site. However, there have been no studies to assess the diagnostic value of NBI in Dieulafoy's disease.

Pathological examination. Pathology or autopsy pathology presents with arterial malformation in the bronchial submucosa. The tortuous, dilated, deformed artery forms small nodules coated with bronchial mucosa, protrudes from the bronchial lumen, and is only a few millimeters in diameter and height. In some cases, deformed blood vessels have an opening within the bronchial lumen, or the diseased bronchus is surrounded by rich blood vessels, some of which invade the bronchial wall and directly reach the submucosa (5). A diagnosis of Dieulafoy's disease depends on the results of pathological examination. However, since this can easily lead to massive bleeding, its use has been limited. Therefore, the incidence of Dieulafoy's disease may have been underestimated.

\section{Differential diagnosis}

Dieulafoy's disease and endobronchial hemorrhagic lesions are primarily distinguished by differential diagnosis. Additionally, the disease must be distinguished from early endobronchial cancer to avoid misdiagnosis.

Bronchial arteriovenous malformation (46). Bronchial arteriovenous malformation can manifest as an endobronchial vascular lesion. The presence of abnormal blood vessels in the lesion can be demonstrated by EBUS or NBI, but it is difficult to distinguish via bronchoscopy. Bronchial angiography can be used to clarify the communication between the bronchial artery and the pulmonary circulation or cavernous hemangioma.

Bronchial artery aneurysm (47). Bronchial artery aneurysm can manifest as an endobronchial vascular lesion. This condition can be distinguished from Dieulafoy's disease by bronchial angiography or multi-slide CT angiography as an aneurysm-like dilation of the bronchial artery.

Lobular capillary hemangioma (48). This condition has no typical symptoms other than hemoptysis. Intraluminal neoplasms can be seen under bronchoscopy. Ulcers and bleeding are visible on the surface and are difficult to distinguish from Dieulafoy's disease of the bronchus. The differential diagnosis primarily relies on pathological examination.

Tracheal capillary hemangioma (49). In general, tracheal capillary hemangioma is similar to tracheal lobular capillary hemangioma and Dieulafoy's disease of the bronchus in terms of clinical manifestations, laboratory examination results, 
imaging and endoscopy. The differential diagnosis primarily relies on pathological examination.

Early cancer. If Dieulafoy's disease is suspected based on bronchoscopy, and the possibility of early cancer cannot be ruled out, NBI and EBUS may be used to determine the presence of thickened and abnormally tortuous and disordered vessels within the lesion. Fluorescence bronchoscopy preliminarily determines the malignancy of the lesion.

\section{Treatment and prognosis}

Existing methods for the treatment of Dieulafoy's disease of the bronchus include conservative internal medication, selective bronchial artery embolization (SBAE), pulmonary lobectomy and argon plasma coagulation via bronchoscopy. Currently, SBAE is the preferred surgical approach, and lobectomy of the diseased lobe is used following embolization failure or recurrent post-embolization hemoptysis. Only one case of argon plasma coagulation via bronchoscopy has been reported (24).

Medication. As the condition presents with bleeding caused by the rupture of the bronchial or pulmonary artery, internal treatment with hemostatic agents often has poor efficacy for Dieulafoy's disease of the bronchus (39). However, for individual patients, pituitrin and thrombin may occasionally demonstrate good therapeutic effects (30).

Bronchoscopic treatment. Topical application of hemostatic drugs under bronchoscopy also has poor efficacy. To date, only Dalar et al (24) has reported the success of treating one patient with Dieulafoy's disease of the bronchus by argon plasma coagulation via bronchoscopy. However, the patient underwent no bronchial angiography or pathological diagnosis, and Dieulafoy's disease of the bronchus was diagnosed based solely on the presentations under bronchoscopy. Thus, the diagnosis of that patient remains controversial. In Dieulafoy's disease, once blood vessels rupture and bleed, the hemorrhage is fast and massive, resulting in an unclear field of view under bronchoscopy. Hence, it is our belief that bleeding from Dieulafoy's disease is not suited to dotted electrocoagulation and superficial hemostasis by argon-beam-coagulator burning, laser treatment and freezing under bronchoscopy. Nevertheless, this approach is feasible to remove blood clots that block the bronchial lumen and to clarify the bleeding site under bronchoscopy, thereby determining the lesion site for bronchial angiography or surgery. The use of a Dumon silicone stent for compression was reported in a patient with Dieulafoy's disease of the left main bronchus, and the patient was followed up for 8 months without recurrent hemorrhage (44). This study indicated that hemostasis by stent compression may be an alternative treatment option. Additionally, a bronchoscopic balloon can be used to compress the bronchus at the bleeding site to provide preparation time for SBAE. For the treatment of gastrointestinal Dieulafoy's disease, coagulant injection under digestive endoscopy can be used for hemostasis. However, no study has reported the use of this method for Dieulafoy's disease of the bronchus, thus the feasibility, safety and efficacy of the method are currently undetermined.
$S B A E$. The SBAE procedure is effective in most patients with Dieulafoy's disease of the bronchus; however, hemoptysis may recur after surgery. Bhatia et al (10) reported the case of one patient with recurrent hemorrhage who had undergone SBAE seven times. Patients with abnormal blood vessels from the pulmonary artery are often non-responsive to SBAE. In our previous repor (39), all six patients with Dieulafoy's disease of the bronchus underwent SBAE at the corresponding site of hemorrhage. Hemorrhage was stopped in one patient following SBAE, who then underwent a pulmonary lobectomy; the other five patients all underwent SBAE and were followed up for 1 to 5 years. Furthermore, hemoptysis was not recurrent in four of the patients, though one patient did experience relapse.

Surgical treatment. In cases of SBAE failure, SBAE for hemoptysis relapse or no SBAE treatment, a lobectomy may be performed at the corresponding site of the lesion. Hemoptysis is unlikely to recur following resection of the diseased lung lobe. In a report by Yang et al (26), 13 patients underwent lobectomy of the diseased lobe; 12 did not experience recurrent hemoptysis, and one died due to hemorrhage after bronchoscopic biopsy, but not due to surgery.

\section{Conclusions}

Dieulafoy's disease of the bronchus lacks specificity in clinical symptoms and primarily presents as massive hemoptysis, although other respiratory or cardiovascular symptoms may also occur. Due to its relative rarity, respiratory physicians currently lack sufficient awareness of Dieulafoy's disease of the bronchus, thus the disease is likely to be missed or misdiagnosed. Serious consequences may result if attention is not paid to the diagnosis and treatment process. In particular, when local protrusion changes are found in the lumen during routine bronchoscopy, physicians perform routine biopsies which can cause fatal bleeding. Therefore, to rule out the possibility of Dieulafoy's disease of the bronchus, bronchial angiography, multi-slice CT angiography, EBUS and NBI examinations must be considered for patients undergoing bronchoscopy who present with cryptogenic hemoptysis and/or smooth protrusion within the bronchial lumen resembling Dieulafoy's disease (even those who do not experience hemoptysis). Biopsy must be prohibited if bronchial angiography demonstrates: i) The presence of a bronchial artery with abnormal tortuosity and dilation, as well as rupture and bleeding at the lesion site; ii) an enhanced CT value $>100$ for the lesion; or iii) EBUS and NBI results of abnormal blood flow within the lesion. Caution must be taken during bronchoscopic operations, such as biopsy and brushing, to prevent asphyxial hemorrhage. At present, SBAE and pulmonary lobectomy are the primary treatment methods for Dieulafoy's disease of the bronchus, although conservative drug treatment and flexible bronchoscope argon plasma coagulation have also been successfully used. SBAE can retain part of the function of the diseased lung, but may result in relapse following treatment. Lobectomy is a radical cure for Dieulafoy's disease, although complete removal of the diseased lung may affect the patient's quality of life. Thus, clinicians must assess the advantages and disadvantages, and select the most appropriate treatment method depending on the physical manifestations of each patient. 


\section{Acknowledgements}

Not applicable.

\section{Funding}

The present study was supported by the Special and Joint Program of the Yunnan Provincial Science and Technology Department and Kunming Medical University [grant no. 2018FE001(-206)], the Yunnan Health Training Project of High Level Talents (grant no. and H-2018095), the Young Academic and Technical Leaders of Yunnan Province (grant no. 2017HB053), the Science and Technology Program for Public Wellbeing of Yunnan Province (grant no. 2014RA020). and the Famous Doctors of High-level Talent Training Support Program of Yunnan Province (grant nos. YNWR-MY-2020-013 and YNWR-MY-2020-027).

\section{Availability of data and materials}

Not applicable.

\section{Authors' contributions}

$\mathrm{XX}$ and JY were the primary contributors to the writing of the manuscript. SX and JL contributed to the diagnosis and differential diagnosis of bronchial Dieulafoy's disease. YD designed the study and made important revisions to the manuscript. Data authentication is not applicable. All authors read and approved the final manuscript.

\section{Ethics approval and consent to participate}

Not applicable.

\section{Patient consent for publication}

The patients provided consent for publication of the figures included in the manuscript.

\section{Competing interests}

The authors declare that they have no competing interests.

\section{References}

1. Dieulafoy G: Exulceratio simplex: Surgical intervention in overwhelming haematemesis following simple exulceration of the stomach Bull Acad Med 49: 49-84, 1898.

2. Sweerts M, Nicholson AG, Goldstraw P and Corrin B Dieulafoy's disease of the bronchus. Thorax 50: 697-698, 1995.

3. Qian X, Du Q, Wei N, Wang M, Wang H and Tang Y: Bronchial Dieulafoy's disease: A retrospective analysis of 73 cases. BMC Pulm Med 19: 104, 2019.

4. Smith B, Hart D and Alam N: Dieulafoy's disease of the bronchus: A rare cause of massive hemoptysis. Respirol Case Rep 2: 55-56, 2014

5. Pomplun S and Sheaff MT: Dieulafoy's disease of the bronchus: An uncommon entity. Histopathology 46: 598-599, 2005.

6. Barisione EE, Ferretti GG, Ravera SS and Salio MM: Dieulafoy's disease of the bronchus: A possible mistake. Multidiscip Respir Med 7: 40, 2012.
7. Parrot A, Antoine M, Khalil A, Théodore J, Mangiapan G, Bazelly $\mathrm{B}$ and Fartoukh M: Approach to diagnosis and pathological examination in bronchial Dieulafoy disease: A case series. Respir Res 9: $58,2008$.

8. Sheth HS, Maldonado F and Lentz RJ: Two cases of Dieulafoy lesions of the bronchus with novel comorbid associations and endobronchial ablative management. Medicine (Baltimore) 97: e9754, 2018.

9. Hope-Gill B and Prathibha BV: Bronchoscopic and angiographic findings in Dieulafoy's disease of the bronchus. Hosp Med 63: 178-179, 2002.

10. Bhatia P, Hendy MS, Li-Kam-Wa E and Bowyer PK: Recurrent embolotherapy in Dieulafoy's disease of the bronchus. Can Respir J 10: 331-333, 2003

11. Kuzucu A, Gürses I, Soysal O, Kutlu R and Ozgel M: Dieulafoy's disease: A cause of massive hemoptysis that is probably underdiagnosed. Ann Thorac Surg 80: 1126-1128, 2005.

12. Pan F, Wang F, Liu Z, Yuan F, Sun KK, Gao ZC and Sun Y: The computed tomography angiography features of Dieulafoy disease of the bronchu]. Zhonghua Jie He He Hu Xi Za Zhi 41: 949-953, 2018 (In Chinese).

13. Gharagozloo F, Rennert D, Margolis M, Tempesta B, Schwartz A, Cole V and Wang KP: Dieulafoy lesion of the bronchus: Review of the literature and report of the 13th case. J Bronchol 15: 38-40, 2008.

14. Zhou P, Yu W, Chen K, Li X and Xia Q: A case report and review of literature of Dieulafoy's disease of bronchus: A rare life-threatening pathologic vascular condition. Medicine (Baltimore) 98: e14471, 2019.

15. Trisolini R, Cancellieri A and Patelli M: Life-threatening bleeding after endobronchial biopsy in a patient with bronchiectasis. Am J Respir Crit Care Med 188: e9-e10, 2013.

16. van der Werf TS, Timmer A and Zijlstra JG: Fatal haemorrhage from Dieulafoy's disease of the bronchus. Thorax 54: 184-185, 1999.

17. Stoopen E, Baquera-Heredia J, Cortes D and Green L: Dieulafoy's disease of the bronchus in association with a paravertebral neurilemoma. Chest 119: 292-294, 2001.

18. Löschhorn C, Nierhoff N, Mayer R, Zaunbauer W, Neuweiler J and Knoblauch A: Dieulafoy's disease of the lung: A potential disaster for the bronchoscopist. Respiration 73: 562-565, 2006.

19. Savale L, Parrot A, Khalil A, Antoine M, Théodore J, Carette MF, Mayaud C and Fartoukh M: Cryptogenic hemoptysis: From a benign to a life-threatening pathologic vascular condition. Am J Respir Crit Care Med 175: 1181-1185, 2007.

20. D'Souza F and Sharma R: Dieulafoy's disease of the bronchus. Pathology 42: 683-684, 2010.

21. Gurioli C, Casoni GL, Gurioli C, Tomassetti S, Romagnoli M, Ravaglia C and Poletti V: Endobronchial ultrasound in Dieulafoy's disease of the bronchus: An additional application of EBUS. Monaldi Arch Chest Dis 73: 166-168, 2010.

22. Kolb T, Gilbert C, Fishman EK, Terry P, Pearse D, Feller-Kopman D and Yarmus L: Dieulafoy's disease of the bronchus. Am J Respir Crit Care Med 186: 1191, 2012.

23. Fang Y, Wu Q and Wang B: Dieulafoy's disease of the bronchus: Report of a case and review of the literature. J Cardiothorac Surg 9: 191, 2014.

24. Dalar L, Sökücü SN, Özdemir C, Büyükkale S and Altın S: Endobronchial argon plasma coagulation for treatment of Dieulafoy disease. Respir Care 60: e11-e13, 2015.

25. Xie BS, Chen YS, Lin MF, Huang QH and Lin ZS: Dieulafoy's disease of the bronchus: A case report and review of the literature. Zhonghua Jie He He Hu Xi Za Zhi 29: 801-803, 2006 (In Chinese).

26. Yang RH, Li JF, Liu J, Sun KK, Cao ZL and Gao ZC: Dieulafoy disease of the bronchus: 3 cases report with literature review. Zhonghua Jie He He Hu Xi Za Zhi 36: 577-580, 2013 (In Chinese).

27. Liu FL, Chen EG, Zhou P, Jin M, Yang L and Ying KJ: Tracheal lobular capillary hemangioma: Two case report and review of the literature. Zhonghua Jie He He Hu Xi Za Zhi 33: 849-852, 2010 (In Chinese).

28. Hadjiphilippou S, Shah PL, Rice A, Padley S and Hind M: Bronchial dieulafoy lesion. A 20-year history of unexplained hemoptysis. Am J Respir Crit Care Med 195: 397, 2017.

29. Madan K, Dhungana A, Hadda V, Mohan A and Guleria R: Flexible bronchoscopic argon plasma coagulation for management of massive hemoptysis in bronchial Dieulafoy's disease. Lung India 34: 99-101, 2017.

30. Niu HL, Yi P, Wang H, Wang FH, Liu W, Gao Q, Chen ZR, Xia JQ and Zeng RX: Infantile Dieulafoy's disease of bronchus: Report of a case. Zhonghua Bing Li Xue Za Zhi 46: 731-732, 2017 (In Chinese).

31. Wadji MB and Farahzadi A: Dieulafoy's disease of the bronchial tree: A case report. Sao Paulo Med J 135: 396-400, 2017. 
32. Yang D, Rong C, Gu J, Xu L, Zhang J, Zhang G and Shen C: Dieulafoy disease of the trachea with recurrent episodes of massive hemoptysis: A case report. Medicine (Baltimore) 96: e5855, 2017.

33. Bonnefoy V, Garnier M, Tavolaro S, Antoine M, Assouad J, Fartoukh M and Gibelin A: Bronchial Dieulafoy's disease: Visualization of embolization particles in bronchial aspirate. Am J Respir Crit Care Med 198: 954-955, 2018.

34. Mincholé E, Penin RM and Rosell A: The utility of linear endobronchial ultrasound for the incidental finding of Dieulafoy disease of the bronchus. J Bronchology Interv Pulmonol 25: e48-e50, 2018.

35. Wang F, Kuang TG, Wang JF and Yang YH: A rare cause of recurrent fatal hemoptysis: Dieulafoy's disease of the bronchus. Chin Med J (Engl) 131: 2758-2759, 2018.

36. Chen W, Chen P, Li X, Gao X and Li J: Clinical characteristics and treatments for bronchial Dieulafoy's disease. Respir Med Case Rep 26: 229-235, 2019.

37. Tang P, Wu T, Li C, Lv C, Huang J, Deng Z and Ding Q: Dieulafoy disease of the bronchus involving bilateral arteries: A case report and literature review. Medicine (Baltimore) 98: e17798, 2019.

38. White C, Ottaviano P, Munn N, Shweihat Y and Zeid F: Massive hemoptysis due to recurrence of bronchial to pulmonary vascular malformation: A case report. Respir Med Case Rep 26: 248-250, 2019.

39. Liu Y, Li Y, Xing X, Xiao Y, Li Z, Yang Y and Wu X: Diagnosis and treatment of Dieulafoy's disease of the bronchus. China J Endosc 20: 795-799, 2014.

40. Xia XD, Ye LP, Zhang WX, Wu CY, Yan SS, Weng HX, Lin J, Xu H, Zhang YF, Dai YR, et al: Massive cryptogenic hemoptysis undergoing pulmonary resection: Clinical and pathological characteristics and management. Int J Clin Exp Med 8: 18130-18136, 2015.

41. Padilla-Serrano A, Estrella-Palomares V, Martinez-Palacios B and Gonzalez-Spinola J: A case of massive hemoptysis related to a smoking-history: An acquired form of the Dieulafoy's disease? Rev Port Pneumol 21: 276-279, 2015.
42. Antoune M, Maniacal G, Bazelly B, et al: Dieulafoy's vascular malformation of the bronchus report of 3 cases. In: 1998 Annual Meeting of the United States and Canada Academy of Pathology 11: 171, 1998

43. Rennert D, Gharagozloo F, Schwartz AM, Margolis M, Tempesta B and Wu J: Dieulafoy's lesion of the bronchus: Report of a case and review of the literature. Pathol Case Rev 12: 93-95, 2007.

44. Fields EL and De Keratry DR: Dieulafoy disease of the bronchus: Case report and presentation of a novel therapeutic modality. J Bronchology Interv Pulmonol 15: 107-109, 2008.

45. Wang T, Zhang J, Wang Y, Pei Y, Xu M and Zhang C: Diagnosis of bronchial artery - pulmonary artery fistula by the combination of narrow band imaging with bronchial arteriography: A case report. Zhonghua Jie He He Hu Xi Za Zhi 38: 148-149, 2015.

46. Sharifi M, Messersmith R, Newman B, Chung Y and Lakier JB: Bronchial arteriovenous malformation in a child with hemoptysis. A case report. Angiology 47: 203-209, 1996.

47. Cerezo Lajas A, Rodríguez Guzmán MDC and de Miguel Díez J: Left Bronchial Artery Aneurysm. Arch Bronconeumol 55: 215 , 2019 (In English, Spanish).

48. Dermawan JK, Ko JS and Billings SD: Intravascular Lobular Capillary Hemangioma (Intravascular Pyogenic Granuloma): A Clinicopathologic Study of 40 Cases. Am J Surg Pathol: Jun 2,2020 (Epub ahead of Print). doi: 10.1097/PAS.0000000000001509.

49. Özgül MA, Tanrıverdi E, Gül Ș, Asuk ZY, Acat M, Abbaslı K, Fener NA and Çetinkaya E: A Rare Cause of Hemoptysis in Childhood: Tracheal Capillary Hemangioma. Turk Thorac J 18: 131-133, 2017.

This work is licensed under a Creative Commons Attribution-NonCommercial-NoDerivatives 4.0 International (CC BY-NC-ND 4.0) License. 